\title{
Ophiobolin O Isolated from Aspergillus ustus Induces G1 Arrest of MCF-7 Cells through Interaction with AKT/GSK3ß/Cyclin D1 Signaling
}

\section{Cuiting Lv ${ }^{1, \dagger}$, Wenxing Qin ${ }^{2, \dagger}$, Tonghan Zhu ${ }^{3, \dagger}$, Shanjian Wei ${ }^{1}$, Kui Hong ${ }^{4, *}$, Weiming Zhu ${ }^{3, *}$, Ruohua Chen ${ }^{5, *}$ and Caiguo Huang ${ }^{1, *}$}

1 Department of Biochemistry and Molecular Biology, College of Basic Medical Second Military Medical University, 800 Xiangyin Road, Shanghai 200433, China; E-Mails: lvcuiting961021@126.com (C.L.); sjwei8012@hotmail.com (S.W.)

2 Teaching Management Department, Yangpu Hospital, Tongji University School of Medicine, 450 Tengyue Road, Shanghai 200090, China; E-Mail: wendy_yes1@sina.com

3 Key Laboratory of Marine Drugs, Ministry of Education of China, School of Medicine and Pharmacy, Ocean University of China, Qingdao 266003, China; E-Mail: sdueduzth@126.com

4 Key Laboratory of Combinatorial Biosynthesis and Drug Discovery, Ministry of Education, School of Pharmaceutical Sciences, Wuhan University, Wuhan 430071, China

5 VIP Medicine Department, Changhai Hospital, Shanghai 200433, China

$\dagger$ These authors contributed equally to this work.

* Author to whom correspondence should be addressed; E-Mails: kuihong31@whu.edu.cn (K.H.); weimingzhu@ouc.edu.cn (W.Z.); rhchen1964@sohu.com (R.C.); huangcaig@hotmail.com (C.H.); Tel./Fax: +86-27-68752442 (K.H.); +86-532-82031268 (W.Z.); +86-21-3111-6666 (R.C.); Tel.: +86-21-8187-0970 (ext. 8020) (C.H.); Fax: +86-21-6533-4344 (C.H.).

Academic Editor: Keith Glaser

Received: 9 May 2014 / Accepted: 26 November 2014 / Published: 16 January 2015

\begin{abstract}
Ophiobolin $\mathrm{O}$ is a member of ophiobolin family, which has been proved to be a potent anti-tumor drug candidate for human breast cancer. However, the anti-tumor effect and the mechanism of ophiobolin $\mathrm{O}$ remain unclear. In this study, we further verified ophiobolin O-induced G1 phase arrest in human breast cancer MCF-7 cells, and found that ophiobolin $O$ reduced the phosphorylation level of AKT and GSK3 $\beta$, and induced down-regulation of cyclin D1. The inverse docking (INVDOCK) analysis indicated that ophiobolin O could bind to GSK3 $\beta$, and GSK3 $\beta$ knockdown abolished cyclin D1 degradation and G1 phase arrest. Pre-treatment with phosphatase inhibitor sodium or thovanadate halted dephosphorylation
\end{abstract}


of AKT and GSK3 $\beta$, and blocked ophiobolin O-induced G1 phase arrest. These data suggest that ophiobolin O may induce G1 arrest in MCF-7 cells through interaction with AKT/GSK3 $\beta /$ cyclin D1 signaling. In vivo, ophiobolin $\mathrm{O}$ suppressed tumor growth and showed little toxicity in mouse xenograft models. Overall, these findings provide theoretical basis for the therapeutic use of ophiobolin $\mathrm{O}$.

Keywords: ophiobolin O; G1 arrest; AKT/GSK3ß/cyclin D1 signaling

\section{Introduction}

Ophiobolins belong to the family of natural sesquiterpenes that are characterized by a tricyclic (5-7-5) ring system. Ophiobolins as secondary metabolites that are produced by pathogenic fungi, show a broad spectrum of inhibitory activity against nematodes, fungi, bacteria and cancer cells [1-3]. Ophiobolin O (Figure 1A) is the member of ophiobolin family, which we have proved is a potent anti-tumor drug candidate for human breast cancer. The ophiobolin $\mathrm{O}$ we used is a natural compound that have been isolated from Aspergillus ustus 094102 [4].

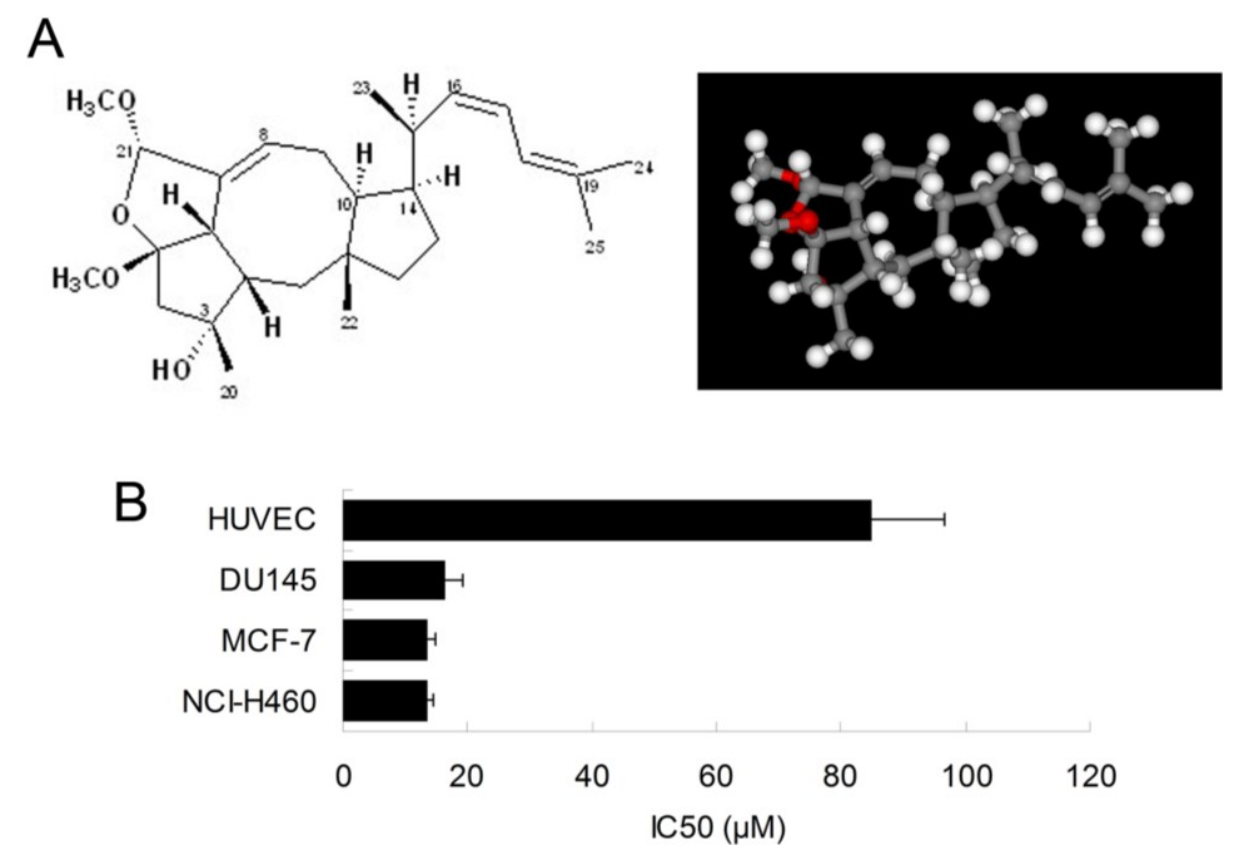

Figure 1. Inhibitory effect of ophiobolin O in human cancer cells. (A) Chemical structure of ophiobolin O (left); and energy-minimized 3D structure of ophiobolin O (right); (B) IC50 of ophiobolin O on viability of HUVEC, DU145 MCF-7 and NCI-H460cell lines. Cells were treated with ophiobolin $\mathrm{O}$ atvarious concentrations for $48 \mathrm{~h}$ and processed for MTT assay.

In previous study, we found that the low concentration of ophiobolin $\mathrm{O}(0.1 \mu \mathrm{M})$ down-regulated the expression of resistance-related protein P-Glycoprotein (P-gp, also known as MDR1), which makes Adriamycin-resistant human breast carcinoma (MCF-7/ADR) cells more sensitive to Adriamycin treatment. Therefore, we demonstrated that ophiobolin $\mathrm{O}$ reversed Adriamycin resistance, and suggested ophiobolin $\mathrm{O}$ acted as a potential agent to reverse chemotherapy drug resistance [5]. On the other hand, 
ophiobolin O reduced the viability of human breast cancer MCF-7 cells in dose-dependent manner and efficiently induced apoptosis and cell cycle arrest. We further proved ophiobolin O-induced cell apoptosis was regulated via activation of MAPK signaling pathways. However, the anti-tumor effect and the mechanism of ophiobolin O remain unclear, since we just used one method to detect G1 phase arrest, which lacking of test and verification; and we also did not figure out about the mechanism of ophiobolin O-induced cell cycle arrest at that time [4].

In the current study, we further verified ophiobolin O-induced G1 phase arrest in MCF-7 cells. Then we investigated the putative protein targets of ophiobolin $\mathrm{O}$ using INVDOCK program. Three apoptotic or G1 phase arrest-related proteins were extracted. Two of them were closely related to cell apoptosis, and could validate our previous study [4]. The rest one was GSK3 $\beta$, which is an upstream regulator of G1 checkpoint [6]. In order to check whether GSK3 $\beta$ is involved in ophiobolin O-induced G1 phase arrest, we detected the protein level and knocked down GSK3 $\beta$ expression using siRNA. Then we found that GSK3 $\beta$ knocked-down cells were not sensitive to ophiobolin O anymore, suggesting that ophiobolin O may target GSK3 $\beta$ to induced G1 phase arrest and growth inhibition in MCF-7 cells. Furthermore, ophiobolin $\mathrm{O}$ also induced dephosphorylation of AKT, and pre-treatment with phosphatase inhibitor sodium orthovanadate halted dephosphorylation of AKT and GSK3 $\beta$, and blocked ophiobolin O-induced G1 phase arrest. Therefore, the anti-proliferative effect of ophiobolin O might be linked with G1 to S phase arrest, which was mediated by the Akt/GSK3ß/cyclin D1 pathway. Finally, ophiobolin O could suppress tumorigenesis in the xenograft mouse model, suggesting that ophiobolin $\mathrm{O}$ suppresses tumor growth in breast cancer.

\section{Results and Discussion}

\subsection{Ophiobolin O Inhibits the Proliferation of MCF-7 Cells}

We have previously shown that the natural compound ophiobolin O isolated from Aspergillus ustus 094102 inhibited the growth of human breast cancer cells [4]. In this study, we further examined the inhibitory effect of ophiobolin O on proliferation of other cell lines, such as HUVEC (Human umbilical vein endothelial cell line), DU145 (Human prostate cancer cell line) and NCI-H460 (Human large cell lung carcinoma cell line). The results demonstrated that breast cancer cells MCF-7 was significantly sensitive to ophiobolin $\mathrm{O}$, with IC 50 value of $13.45 \pm 1.26 \mu \mathrm{M}$. The $\mathrm{IC}_{50}$ values were $84.96 \pm 11.73 \mu \mathrm{M}$, $16.48 \pm 2.68 \mu \mathrm{M}$ and $13.55 \pm 1.01 \mu \mathrm{M}$ when ophiobolin O treated HUVEC, DU145 and NCI-H460 cells, respectively. It is worth noting that normal human umbilical vein endothelial cells (HUVEC) are less sensitive to ophiobolin O compared with other cancer cell lines (Figure 1B), suggesting that ophiobolin O has a potent anti-cancer activity that preferentially kills cancer cells, especially breast cancer cells, over normal cells. This selectivity led us to question the mechanism by which ophiobolin $\mathrm{O}$ exerted its effect on MCF-7 cells.

\subsection{Ophiobolin O Induces G1 Arrest in MCF-7 Cells}

In order to test whether the inhibition effect of ophiobolin $\mathrm{O}$ was related to cell cycle progression, cell cycle distribution and related checkpoint factors were studied. MCF-7 cells were treated with $15 \mu \mathrm{M}$ ophiobolin $\mathrm{O}$ for $12 \mathrm{~h}, 24 \mathrm{~h}$ and $48 \mathrm{~h}$, respectively. The results showed that cells treated with 
ophiobolin $\mathrm{O}$ accumulated progressively in $\mathrm{G} 1$ phase (Figure 2A,B). Compared with the negative control, treatment with ophiobolin $\mathrm{O}$ resulted in a significant increase in the proportion of G1 phase cells (control: $40.19 \% \pm 1.03 \% ; 12 \mathrm{~h}: 46.09 \% \pm 5.79 \% ; 24 \mathrm{~h}: 56.18 \% \pm 2.19 \% ; 48 \mathrm{~h}: 72.56 \% \pm 1.35 \%$ ) and about a 1.8-fold increase after $48 \mathrm{~h}$. Meanwhile, G2/M reduced appreciably from $32.57 \% \pm 0.69 \%$ to $5.53 \% \pm 0.88 \%$ during $48 \mathrm{~h}$ incubation. These results suggest that $\mathrm{G} 1$ phase arrest accounts for the anti-proliferative effect of ophiobolin $\mathrm{O}$ observed in MCF-7 cells. Besides, ophiobolin $\mathrm{O}$ treatment resulted in a time-dependent decrease in the protein expression of cyclin D1, cyclin E, CDK2 and increase of p-cyclin D1 (Thr286), p21 and p27. However, the protein levels of CDK4 and CDK6 were not significantly changed during ophiobolin $\mathrm{O}$ treatment (Figure 2b).
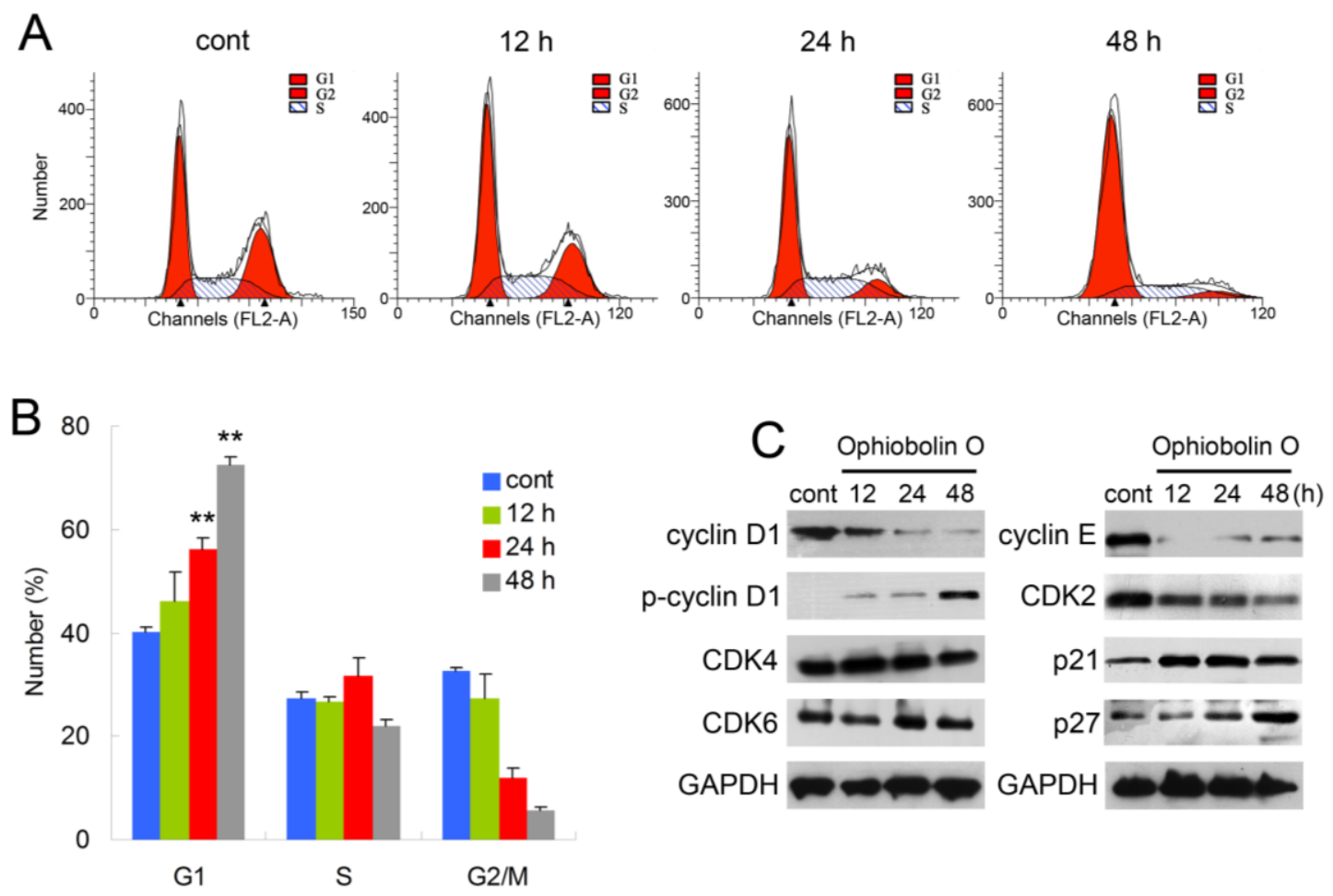

Figure 2. Ophiobolin O induces cell cycle arrest in MCF-7 cells. (A) Ophiobolin O caused cell cycle arrest at the G1 phase. Cells were treated with vehicle and $15 \mu \mathrm{M}$ Ophiobolin $\mathrm{O}$ for 12, 24, and $48 \mathrm{~h}$, and then cell cycle distribution was assessed using flow cytometry; (B) The percentage of cells in different phases of the cell cycle was represented by a bar diagram. The percentage of cells in each population was shown as mean \pm SD from three independent experiments. $* p<0.05, * * p<0.01$; (C) Western blot analysis of G1 transition-related proteins were analyzed by Western blotting assay. GAPDH was used as an equal loading control.

\subsection{Putative Protein Targets for Ophiobolin $O$}

Previously, we observed that ophiobolin O-induced cell apoptosis was regulated via activation of MAPK signaling pathways in MCF-7 cells; but for cell cycle arrest, we did not elucidate how the ophiobolin $\mathrm{O}$ induced G1 phase arrest. Furthermore, to establish the anti-cancer mechanism of ophiobolin $\mathrm{O}$, an inverse docking (INVDOCK) analysis was applied to identify the possible targets of ophiobolin O. Using the INVDOCK program, 90 putative proteins were extracted from the Protein Data 
Bank. Of these, three proteins were closely related to apoptosis and G1 phase arrest (Table 1). TNF (Tumor necrosis factor) is related to cell apoptosis [7]; MAP2K1 (Dual specificity mitogen-activated protein kinase kinase 1; ERK), which is a member of MAPKs, has been proved to be activated during the ophiobolin O-induced apoptosis in MCF-7 cells [4]; GSK3 $\beta$ (Glycogen synthase kinase-3 beta)regulates G1 phase transition through Phosphorylation of cyclin D1 which is one of the key regulatory proteins controlling the transition fromG1 to $\mathrm{S}$ phase [8], therefore, in order to study the mechanism of ophiobolin O-induced G1 phase arrest, 3-D structure of GSK3 $\beta$ was chosen to explore its binding interaction with ophiobolin $\mathrm{O}$. The illustration of ophiobolin $\mathrm{O}$ docked to GSK3 $\beta$ using the INVDOCK program is shown in Figure 3. Such an analysis indicated that ophiobolin O could form a reasonable drug-protein complex with GSK3 $\beta$, which provided a hypothesis that ophiobolin O may induce G1 arrest of MCF-7 cells through interaction with GSK3 $\beta /$ cyclin D1 signaling.

Table 1. Apoptotic or G1 phase-related proteins predicted by INVDOCK to bind to ophiobolin O.

\begin{tabular}{|c|c|c|c|c|c|}
\hline Compond & Protein & Gene & Gene ID & Species & $\begin{array}{c}\text { Ligand-Protein } \\
\text { Interaction Energy Value }\end{array}$ \\
\hline \multirow{3}{*}{ Ophiobolin O } & Glycogen synthase kinase-3beta & GSK-3 $\beta$ & 2932 & Human & -35.8 \\
\hline & Tumor necrosis factor & TNF & 7124 & Human & -37.5 \\
\hline & $\begin{array}{c}\text { Dual specificity mitogen-activated } \\
\text { protein kinase kinase } 1\end{array}$ & MAP2K1 & 5604 & Human & -37.6 \\
\hline
\end{tabular}

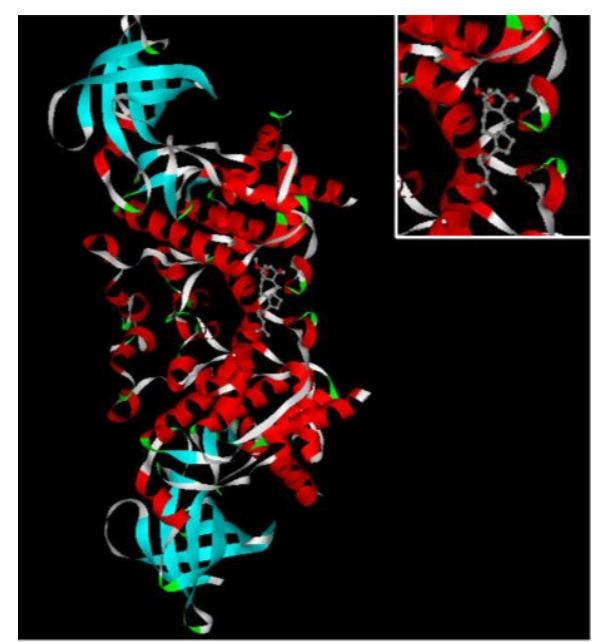

Figure 3. The INVDOCK analysis of ophiobolin O binds to GSK3 $\beta$. Illustration of the ophiobolin $\mathrm{O}$ molecule docked into the GSK3 $\beta$ protein, modeled using the INVDOCK program; upright: the Amplification of binding area.

\subsection{Ophiobolin O Induces G1 Arrest of MCF-7 Cells through Interaction with AKT/GSK3B/Cyclin} D1 Signaling

As described above, it is essential to check whether GSK3 $\beta$ is involved in ophiobolin O-induced G1 phase arrest. Therefore, protein level of GSK $3 \beta$ was analyzed by Western blotting. Furthermore, consider that Akt/GSK3 $\beta$ signaling plays a critical role in cyclin D1 expression and carcinogenesis, we also examined AKT levels. As shown in Figure 4A, ophiobolin $\mathrm{O}$ treatment reduced the phosphorylation 
level of Akt (Ser 473) and GSK3 $\beta$ (Ser 9), indicating an increased activity of GSK3 $\beta$. And GSK3 $\beta$ activity assay proved the increased enzyme activity of GSK3 $\beta$ in vitro (Figure 4B). Furthermore, we used siRNA to knock-down GSK3 $\beta$, and observed that cells transfected with GSK3 $\beta$ siRNA were not sensitive to ophiobolin O anymore: ophiobolin O-induced G1 phase arrest in MCF-7 cells was significantly abolished; and the decrease of cyclin D1 expression was brought to normal levels (Figure 4C,D). Finally, phosphatase inhibitor sodium orthovanadate was applied to halt dephosphorylation of AKT and GSK3 $\beta$, and the pre-treatment also blocked ophiobolin O-induced G1 phase arrest and cyclin D1 reduction.

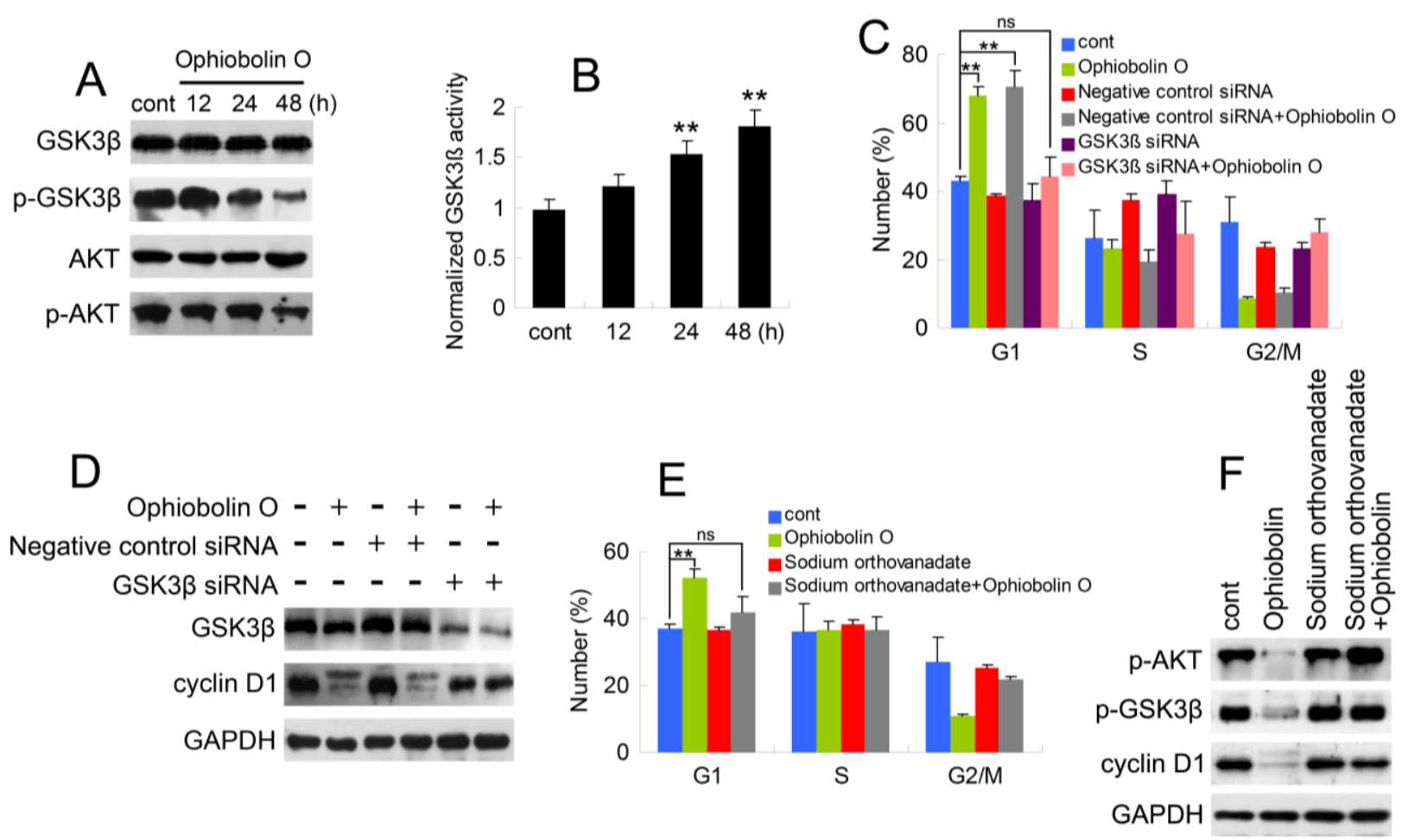

Figure 4. AKT/GSK3 $\beta$ signaling is involved in ophiobolin O-induced G1 phase arrest in MCF-7 cells. (A) GSK3 $\beta$, p-GSK3 $\beta$ (Ser 9), AKT and p-AKT (Ser 473) were detected after ophiobolin O-treatment for $48 \mathrm{~h}$ by western blotting analysis; (B) Statistical results showing the normalized enzyme activities of GSK3 $\beta$. Cells were treated with $15 \mu \mathrm{M}$ ophiobolin $\mathrm{O}$, harvested at $12 \mathrm{~h}, 24 \mathrm{~h}$ and $48 \mathrm{~h}$. GSK3 $\beta$ activity was determined using GSK3 $\beta$ enzyme activity detection kit, and normalized versus control group; (C) Cells transfected with GSK3 $\beta$ siRNA or not were treated with or without $15 \mu \mathrm{M}$ ophiobolin $\mathrm{O}$ for $48 \mathrm{~h}$, then harvested for cell cycle analysis or (D) western blotting assay against GSK3 $\beta$ and cyclin D1. GAPDH was used to ensure equal protein loading; (E) Cells pre-treated with sodium orthovanadate or not were incubated with or without $15 \mu \mathrm{M}$ ophiobolin $\mathrm{O}$ for $48 \mathrm{~h}$, then harvested for cell cycle analysis or (F) western blotting assay against p-AKT, p-GSK3 $\beta$ and cyclin D1. Values were means $\pm \mathrm{SD}$ of three independent experiments. ${ }^{*} p<0.05, * * p<0.01$ versus control group.

Taken together, these results suggest that ophiobolin $\mathrm{O}$ treatment reduces the phosphorylation of Akt and subsequent phosphorylation of GSK3 3 , indicating Akt inactivation and GSK3 $\beta$ activation respectively. This might contribute to cyclinD1 degradation and G1-phase arrest, cause GSK3ßis a critical regulator of cyclin D1 expression $[6,9,10]$. 


\subsection{Ophiobolin O Inhibits Tumor Xenograft Growth}

To evaluate whether ophiobolin O inhibits tumor growth in vivo, MCF-7 cells were injected s.c. into the right armpit of six-week old BALB/c female athymic mice. The injected carcinoma cells grew into palpable tumors in the nude mice within 10 days (Figure 5A). Paclitaxel (PTX)-treated mice $(10 \mathrm{mg} / \mathrm{kg})$ were used as positive control to assess the effect and toxicity of ophiobolin O. In this study, the tumor growth in mice treated with ophiobolin $\mathrm{O}(5,10$, or $20 \mathrm{mg} / \mathrm{kg} / \mathrm{day})$ was dose-dependently slowed (Figure 5B,C). Tumor volume was significantly reduced during ophiobolin $\mathrm{O}$ treatment (Figure 5E). The inhibitory rates at 50th day of 5,10 , and $20 \mathrm{mg} / \mathrm{kg}$ ophiobolin $\mathrm{O}$ treatment group were $34.62 \%, 46.15 \%$, and $69.23 \%$, respectively. The $20 \mathrm{mg} / \mathrm{kg}$ ophiobolin O treatment showed nearly equivalent effect compared to positive control (inhibition rate of $73.08 \%$ ). Furthermore, no significant weight loss was observed during the course of ophiobolin $\mathrm{O}$ treatment, indicating there is feasibility for upward adjustment of dosage for improved treatment outcomes (Figure 5D).

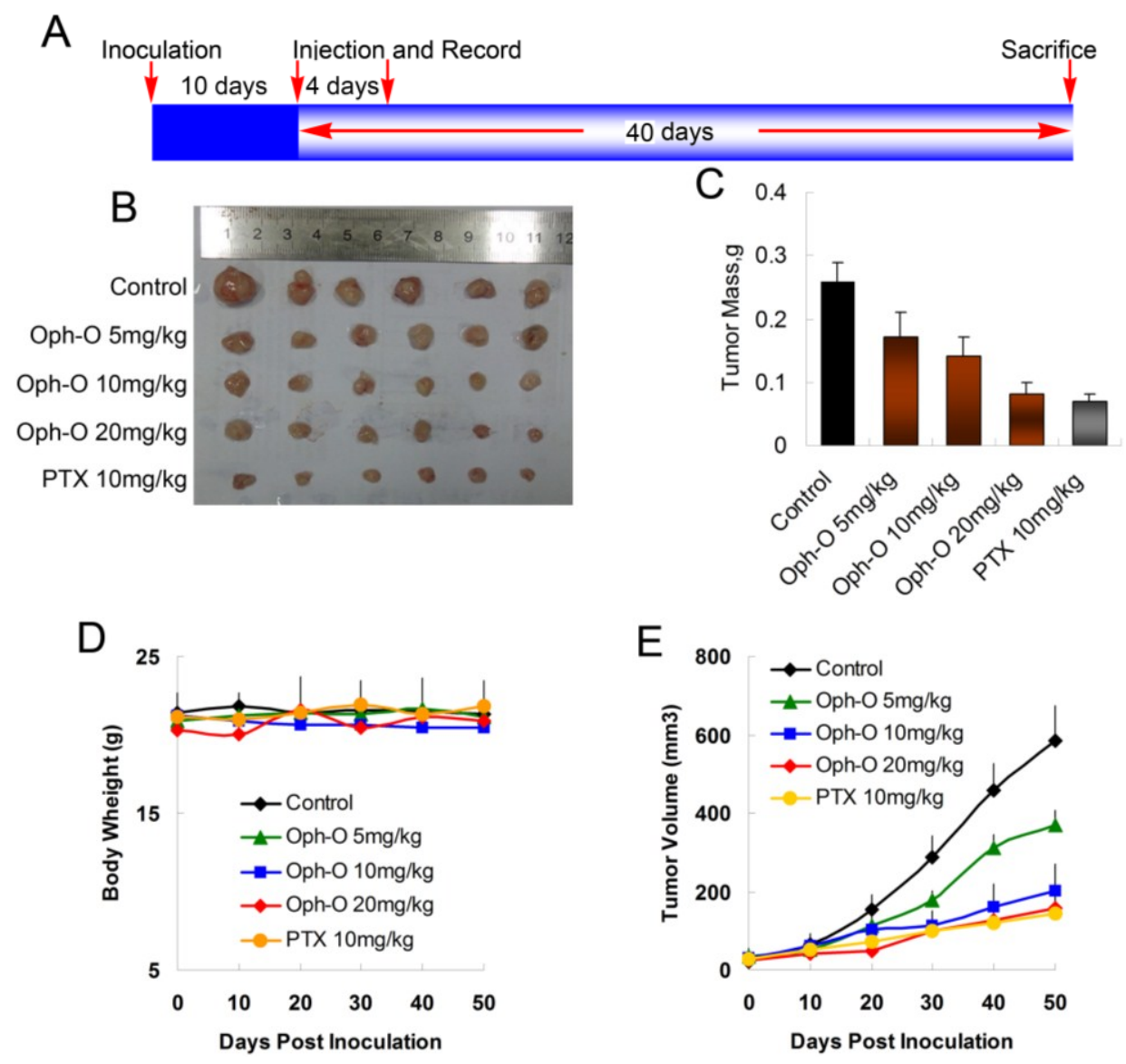

Figure 5. Effect of ophiobolin $\mathrm{O}$ on tumor growth. (A) BALB/c male athymic mice were injected $5 \times 10^{6} \mathrm{MCF}-7$ cells s.c. for the development of subcutaneous tumors. The mice were randomized into five groups and treated with Vehicle (1\% DMSO), $10 \mathrm{mg} / \mathrm{kg}$ PTX or various concentrations of ophiobolin $\mathrm{O}(5,10$, or $20 \mathrm{mg} / \mathrm{kg})$ i.v. every four days until sacrifice according to the protocol in panel (A); (B) Tumor image from various treatment groups; (C) Average tumor mass at sacrifice; (D) Tumor volume measurements; (E) Body weight during the treatment. 


\subsection{Discussion}

So far, studies have shown that ophiobolin $\mathrm{O}$ significantly suppressed tumor growth in vivo and in vitro. It triggered cell cycle arrest at G1 phase through interaction with $\mathrm{AKT} / \mathrm{GSK} 3 \beta /$ cyclin D1 signaling.

In our obvious studies, significant attention was focused on the apoptosis induced by ophiobolin O, and the important role of MAPK signaling pathway involved in apoptotic death in MCF-7 cells. MAPK signaling cascades have been proved to play essential roles in the regulation of a wide variety of cellular processes [11], and several key signaling components and phosphorylation events participate in regulating the cell cycle, apoptosis and even tumorigenesis $[12,13]$. JNK, ERK and p38 are three members of the MAPK family [14]. The JNK pathway is considered to be responsible for the apoptotic response induced by several anti-tumor agents [15], and we have reported that the activation of JNK induced by ophiobolin O triggered apoptosis through the phosphorylation of Bcl-2 in MCF-7 cells. Otherwise, ERK and p38 activation were also observed during ophiobolin $\mathrm{O}$ treatment. The $\mathrm{p} 38^{\mathrm{SAPK} 2}$ and ERK2 have been shown to regulate G1 transition through interaction with cyclin D1 stability by phosphorylating T286 in some reports [16-18]. However, here we declare that AKT/GSK3 $\beta /$ cyclin D1 signaling is responsible for ophiobolin O-induced cyclin D1 degradation and G1 arrest.

In tumor cells, the abnormal molecular activity directly induces the uncontrolled cell proliferation, which is one of the hallmarks of cancer. The majority of human cancers have been reported to have alterations in the function of cell cycle regulatory proteins [19-21]. Cyclin D1 and cyclin E are the key regulatory proteins controlling the transition from G1 to S phase by binding to Cdk4/Cdk6 and CDK2 [12]. Considering the crucial role of cyclin D1, it is not surprising that its expression is down-regulated in cells stimulated with anti-proliferative cytokines. This down-regulation generally manifests at the level of protein stabilization, which was accelerated via the phosphatidylinositol 3-kinase Akt/GSK3 $\beta$ pathway [9]. In normal cells, the levels of cyclin D1 begin to rise early in G1 phase and continue to accumulate until the G1/S-phase boundary when levels rapidly decline [13,14]; glycogen synthase kinase $3 \beta$ (GSK3 $\beta$ ) induces rapid proteolysis of cyclin D1 by phosphorylating cyclin D1 on threonine residue 286 (Thr 286) [8]. Phosphorylation facilitates cyclin D1 nuclear export by the exportin-chromosome maintenance region 1 (CRM1), and the phosphorylated form of the cyclin is subsequently degraded within the cytoplasm [22-24]. In this manner, GSK $3 \beta$ regulates nuclear export and stability of cyclin D1 to ensure the normal mitosis. Usually, GSK3 $\beta$ has a higher level of basal activity in tumor cells than in their normal counterparts [25]. Thus, the inactivation of GSK3 $\beta$ and subsequent up-regulation of cyclin D1 may have a critical role in human cancer cells. In these cells, phosphorylation at Ser 9 by AKT is a major means to inactivate GSK3 $\beta[26,27]$.

In our study, no obvious amount of changes of total GSK3 $\beta$ were observed during the treatment. In contrast, numerous phosphorylation form of GSK3 $\beta$ (Ser 9) was detected in control cells, and showed marked time-dependent decrease after ophiobolin O treatment (Figure 4A), consistently with increase of GSK $3 \beta$ activity (Figure 4B). Therefore, the expression of cyclin D1 reduced and phosphorylated cyclin D1 on threonine residue 286 was subsequently increased (Figure $2 \mathrm{C}$ ). These results indicated that the inactivation of GSK $3 \beta$ contributes to MCF-7 cell proliferation, and GSK3 $\beta$ /cyclin D1 cell signaling maybe involved in ophiobolin O-induced G1 phase arrest. The INVDOCK was designed to confirm the potential targets related with ophiobolin O-induced anti-neoplastic effect, and the results noted that ophiobolin O 
could directly bind to GSK3 $\beta$ (Table 1 and Figure 3). It is accepted that small-molecule drugs generally exert their therapeutic functions by binding to the cavities of proteins to influence their biological activities [28]. This binding may influence phosphorylation of GSK3 $\beta$, therefore activate GSK3 $\beta$ and down-regulate cyclin D1 to induce G1 phase arrest. To investigate weather the putative protein target-GSK3 $\beta$ is responsible for ophiobolin O-induced cyclin D1 degradation and G1 arrest, we used siRNA to knock-down GSK3 $\beta$, and noticed ophiobolin O-induced reduction of cyclin D1 and G1 arrest were significantly abolished (Figure 4C-E). Otherwise, considering GSK3 $\beta$ as a Akt-inactivating factor, we also detected the AKT effect during ophiobolin $\mathrm{O}$ treatment. It appeared that ophiobolin $\mathrm{O}$ inhibited the phosphorylation level of Akt at Ser 473. Furthermore, we used the sodium orthovanadate to up-regulate Akt activity. The sodium orthovanadate is an inhibitor of tyrosine phosphatases, alkaline phosphatases and a number of ATPases; commonly used to improve the activation of protein by inhibiting the dephosphorylation or promoting phosphorylation. It prevents decreased Akt-Ser-473 phosphorylation in the CA1 region, which up-regulates Akt activity [29]. In our study, sodium orthovanadate was applied to halt dephosphorylation of AKT and GSK3 $\beta$, and the pre-treatment also blocked ophiobolin O-induced G1 phase arrest and cyclin D1 reduction. Therefore, we conclude that Ophiobolin O induces G1 arrest of MCF-7 cells through interaction with AKT/GSK3 $/$ cyclin D1 signaling.

All above strongly suggest that ophiobolin $O$ may target AKT/GSK3 $\beta /$ cyclin D1 signaling. The increase of active form of GSK3 $\beta$ accelerates cyclin D1 degradation, and inventually induces G1 phase arrest in MCF-7 cells. Cyclin D1 regulation and the probable mechanisms of ophiobolin O-induced G1 phase arrest are characterized in Figure 6.

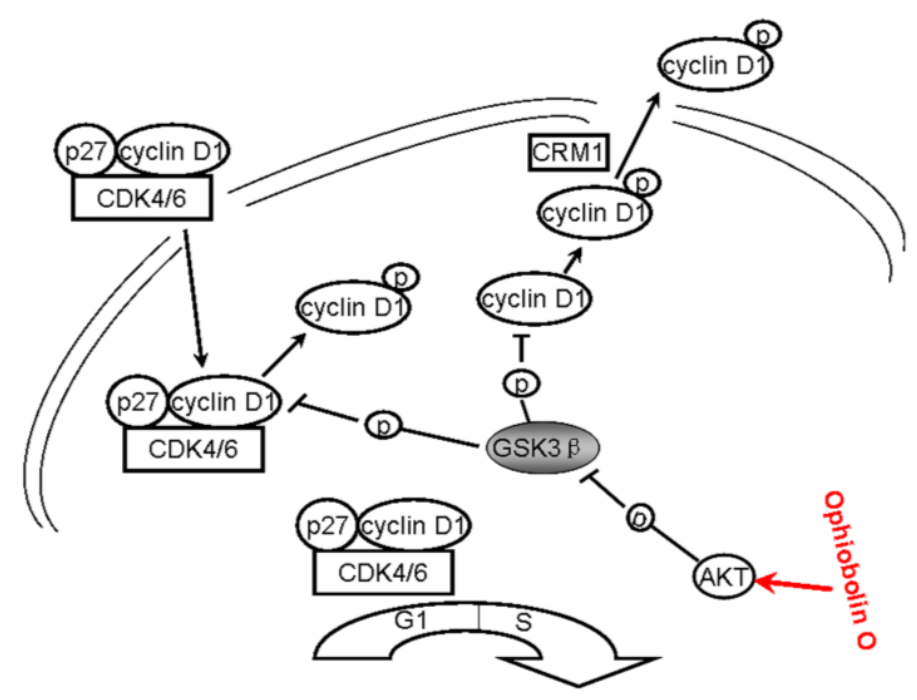

Figure 6. A schematic demonstration for ophiobolin O inducing G1 arrest in MCF-7 cells through interaction with AKT/GSK3 $\beta /$ cyclin D1 signaling.

\section{Experimental Section}

\subsection{Reagents}

DMEM, fetal bovine serum, penicillin and streptomycin were purchased from Biowest (Maine et Loire, France). Cell cycle detection kits were provided by Nanjing KeyGen Biotech Co. Ltd. (Nanjing, China). GAPDH was obtained from Tianjin Sungene Biotech (Tianjin, China). Antibodies against cyclin D1 
(\#2926), p-cyclin D1 (Thr 286, \#3300), GSK3 $\beta$ (\#9315) p-GSK3 $\beta$ (Ser 9, \#4688), CDK4 (\#2906) and CDK6 (\#3136) were purchased from Cell Signaling Technology (Beverly, MA, USA). siRNA used for silence GSK3 $\beta$ gene was purchased from Shanghai Gene Pharma Co., Ltd (Shanghai, China).

\subsection{Chemical Preparation and Cell Culture}

Ophiobolin $\mathrm{O}$ is a natural compound that has been isolated from Aspergillus ustus 094102. The compound was dissolved in $100 \%$ DMSO and stored at $-20^{\circ} \mathrm{C}$, then diluted with cell culture media before using. The final DMSO concentration did not exceed $0.1 \%$. Human breast cancer cell line MCF-7 was purchased from the cell bank of the Shanghai Institute of Cell Biology (Shanghai, China), and was cultured in DMEM with $10 \%$ fetal bovine serum and antibiotics $(100 \mu \mathrm{g} / \mathrm{mL}$ streptomycin and $100 \mathrm{U} / \mathrm{mL}$ penicillin) in a humidified $5 \% \mathrm{CO}_{2}$ incubator at $37^{\circ} \mathrm{C}$.

\subsection{Cell Viability Assay}

Cells were seeded into 96-well plates at $4 \times 104$ cells $/ \mathrm{mL}$, incubated for $24 \mathrm{~h}$, and then treated with the indicated concentrations of ophiobolin $\mathrm{O}(0,2.5,5,10,20,40$ and $80 \mu \mathrm{M})$ for $48 \mathrm{~h}$. Cell viability was determined using MTT assay.

\subsection{Cell Cycle Analysis}

Cells were harvested, washed twice with PBS, fixed in $70 \%$ ethanol and stored at $4{ }^{\circ} \mathrm{C}$ overnight, then washed with PBS, incubated with RNase at $37^{\circ} \mathrm{C}$ for $30 \mathrm{~min}$, and stained with PI $(1 \mathrm{mg} / \mathrm{mL})$ in the presence of RNase A for $30 \mathrm{~min}$. Cell cycle phase analysis was performed by using a FACScalibur flow cytometer.

\subsection{Western Blot Analysis}

Cells were lysed in Western blotting lysis buffer (50 mM Tris, $150 \mathrm{mM} \mathrm{NaCl}, 1 \%$ Triton X-100, 1\% sodium deoxycholate, $0.1 \%$ SDS and $1 \mathrm{mM} \mathrm{PMSF}$ ) at $4{ }^{\circ} \mathrm{C}$ for $30 \mathrm{~min}$. After $12,000 \times g$ centrifugation for $15 \mathrm{~min}$, the protein content of supernatant was quantified using BCA protein assay kit (Beyotime, Shanghai, China). Equal amounts of the protein samples were separated by SDS-PAGE, and transferred to nitrocellulose membranes using an electro-blotting apparatus (Bio-Rad, Hercules, CA, USA). Then the membranes were blocked in blocking buffer (TBST plus 5\% skimmed milk), and incubated with primary antibodies overnight at $4{ }^{\circ} \mathrm{C}$. After that the membranes were washed with TBST and incubated with HRP (horseradish peroxidase)-conjugated secondary antibodies for $1.5 \mathrm{~h}$ at $4{ }^{\circ} \mathrm{C}$. Protein expression was visualized using the chemoluminescence reagent (Millipore, Billerica, MA, USA) and detected on photographic film.

\subsection{Enzyme Activity Assay}

Activity of GSK3 $\beta$ was determined by the GSK3 $\beta$ enzyme activity detection kit (GENMED, Shanghai, China) according to manufacturer's instructions. Briefly, the samples of drug-treated cells were rinsed by reagent $\mathrm{A}$ and then re-suspended with the extraction buffer reagent $\mathrm{B}$. After $30 \mathrm{~min}$ incubation on ice, the mixture was centrifuged at $4{ }^{\circ} \mathrm{C}$. The supernatant was collected and then assayed 
for enzyme activity, by mixing $65 \mu \mathrm{L}$ reagent $\mathrm{C}, 10 \mu \mathrm{L}$ reagent $\mathrm{D}, 10 \mu \mathrm{L}$ reagent $\mathrm{E}$ and $10 \mu \mathrm{L}$ reagent $\mathrm{F}$ and incubation at $30{ }^{\circ} \mathrm{C}$ for $3 \mathrm{~min}$. Immediately after the addition of $5 \mu \mathrm{L}$ supernatant to the reagent mixture, the optical density was measured at $340 \mathrm{~nm}$ using a Microplate Reader at 0 min and $5 \mathrm{~min}$. The activity was measured as the difference between the absorbance value at 0 and $5 \mathrm{~min}$ [30].

\subsection{Identification of Putative Protein Targets for Ophiobolin O}

To verify the proteins related to possible Ophiobolin $\mathrm{O}$ targets, a flexible ligand-protein inverse docking program, INVDOCK was used to identify putative protein targets for Ophiobolin O. The 3D structure of Ophiobolin O was input into the INVDOCK program. The software automatically searched for protein cavities derived from 3D structures of all candidate proteins. An energy value statistically derived from the analysis of a large number of PDB ligand-protein complexes was used as a threshold for screening likely binders. A human protein was considered as a putative target of Ophiobolin $\mathrm{O}$ if the molecule could be docked into the protein and the binding satisfied a molecular-mechanics based criterion for chemical complementarity [31].

\section{8. siRNA Transfection}

Cells were plated in six-well plates, grown for $24 \mathrm{~h}$, then transfected with 80 pmol of siRNA for $12 \mathrm{~h}$ using Lipofectamine 2000 reagent and OPTIMEM reduced serum medium (Invitrogen, Carlsbad, CA, USA). The cells were assayed within $72 \mathrm{~h}$ after transfection.

\subsection{Mouse Xenograft Model}

The mouse xenograft model was established by injection of $5 \times 10^{6}$ cells s.c. into the right armpit of five-week old BALB/c female athymic mice (National Rodent Laboratory Animal Resource, Shanghai, China). The mice were randomized into vehicle control and treatment groups when xenografts were palpable after 10 days. Vehicle or drugs were administered i.v. every four days until sacrifice; body weight and tumor size were measured and recorded at the same time. Tumor size was measured using electronic caliper, and the tumor volumes were calculated using the formula: length $\times$ width $^{2} / 2$. On 50 days, mice were sacrificed; tumors were collected, weighed, and photographed. Tumor inhibition effect was calculated using the following equation: tumor suppression $(\%)=(1-\mathrm{T} / \mathrm{C}) \times 100$, where $\mathrm{T}$ is the average tumor weight of the treated group and $\mathrm{C}$ is that of the control group.

\section{Conclusions}

Ophiobolin O induces G1 phase arrest in human breast cancer MCF-7 cells. It targets GSK3 $\beta$ and interacts with GSK3 $\beta$ /cyclin D1 signaling pathway to induced growth inhibition. In vivo, ophiobolin O suppressed tumor growth and showed little toxcity in mouse xenograft models.

\section{Acknowledgments}

This research project was supported by the National Basic Research Program of China (973 Program) (2010CB833800), by the National Ocean 863 Project of the Ministry of Science and Technology of China (2011AA09070106). 


\section{Author Contributions}

Cuiting Lv: complete the experiment; Wenxing Qin, Caiguo Hang and Ruohua Chen: assist to complete the experiment; Shanjian Wei: data analysis; Tonghan Zhu and Weiming Zhu: the isolation and identification of the ophiobolin O; Kui Hong: the culture of the Aspergillus ustus.

\section{Conflicts of Interest}

The authors declare no conflict of interest.

\section{References}

1. Au, T.K.; Chick, W.S.; Leung, P.C. The biology of ophiobolins. Life Sci. 2000, 67, 733-742.

2. Li, E.; Clark, A.M.; Rotella, D.P.; Hufford, C.D. Microbial metabolites of ophiobolin A and antimicrobial evaluation of ophiobolins. J. Nat. Prod. 1995, 58, 74-81.

3. Arai, M.; Niikawa, H.; Kobayashi, M. Marine-derived fungal sesterterpenes, ophiobolins, inhibit biofilm formation of Mycobacterium species. J. Nat. Med. 2013, 67, 271-275.

4. Yang, T.; Lu, Z.; Meng, L.; Wei, S.; Hong, K.; Zhu, W.; Huang, C. The novel agent ophiobolin O induces apoptosis and cell cycle arrest of MCF-7 cells through activation of MAPK signaling pathways. Bioorg. Med. Chem. Lett. 2012, 22, 579-585.

5. Sun, W.; Lv, C.; Zhu, T.; Yang, X.; Wei, S.; Sun, J.; Hong, K.; Zhu, W.; Huang, C. Ophiobolin-O reverses Adriamycin resistance via cell cycle arrest and apoptosis sensitization in a driamycin-resistant human breast carcinoma (MCF-7/ADR) cells. Mar. Drugs 2013, 11, 4570-4584.

6. Takahashi-Yanaga, F.; Sasaguri, T. GSK-3 beta regulates cyclin D1 expression: A new target for chemotherapy. Cell Signal. 2008, 20, 581-589.

7. Van Antwerp, D.J.; Martin, S.J.; Kafri, T.; Green, D.R.; Verma, I.M. Suppression of TNF-alpha-induced apoptosis by NF-kappaB. Science 1996, 274, 787-789.

8. Diehl, J.A.; Zindy, F.; Sherr, C.J. Inhibition of cyclin D1 phosphorylation on threonine-286 prevents its rapid degradation via the ubiquitin-proteasome pathway. Genes Dev. 1997, 11, 957-972.

9. Diehl, J.A.; Cheng, M.; Roussel, M.F.; Sherr, C.J. Glycogen synthase kinase-3 beta regulates cyclin D1 proteolysis and subcellular localization. Genes Dev. 1998, 12, 3499-3511.

10. Tetsu, O.; McCormick, F. Beta-catenin regulates expression of cyclin D1 incolon carcinoma cells. Nature 1999, 398, 422-426.

11. Zhang, W.; Liu, H.T. MAPK signal pathways in the regulation of cell proliferation in mammalian cells. Cell Res. 2002, 12, 9-18.

12. Miyoshi, N.; Uchida, K.; Osawa, T.; Nakamura, Y. A link between benzyl isothiocyanate-induced cell cycle arrest and apoptosis: Involvement of mitogen-activated protein kinases in the Bcl-2 phosphorylation. Cancer Res. 2004, 64, 2134-2142.

13. Santarpia, L.; Lippman, S.M.; El-Naggar, A.K. Targeting the MAPK-RAS-RAF signaling pathway in cancer therapy. Expert Opin. Ther. Targets 2012, 16, 103-119.

14. Leppä, S.; Bohmann, D. Diverse functions of JNK signaling and c-Jun in stress response and apoptosis. Oncogene 1999, 18, 6158-6162. 
15. Shen, H.M.; Liu, Z.G. JNK signaling pathway is a key modulator in cell death mediated by reactive oxygen and nitrogen species. Free Radic. Biol. Med. 2006, 40, 928-939.

16. Okabe, H.; Lee, S.H.; Phuchareon, J.; Albertson, D.G.; McCormick, F.; Tetsu, O. A critical role for FBXW8 and MAPK in cyclin D1 degradation and cancer cell proliferation. PLoS One 2006, $1, \mathrm{e} 128$.

17. Casanovas, O.; Miro, F.; Estanyol, J.M.; Itarte, E.; Agell, N.; Bachs, O. Osmotic stress regulates the stability of cyclin D1 in ap38 $8^{\mathrm{SAPK}}$-dependent manner. J. Biol. Chem. 2000, 275, 35091-35097.

18. Thoms, H.C.; Dunlop, M.G.; Stark, L.A. p38-mediated inactivation of cyclin D1/cyclin-dependent kinase 4 stimulates nucleolar translocation of RelA and apoptosis in colorectal cancer cells. Cancer Res. 2007, 67, 1660-1669.

19. Sherr, C.J. Cancer cell cycles. Science 1996, 274, 1672-1677.

20. Kastan, M.B.; Bartek, J. Cell-cycle checkpoints and cancer. Nature 2004, 432, 316-323.

21. Malumbres, M.; Barbacid, M.; Cell cycle, CDKs and cancer: A changing paradigm. Nat. Rev. Cancer 2009, 9, 153-166.

22. Alt, J.R.; Cleveland, J.L.; Hannink, M.; Diehl, J.A. Phosphorylation dependent regulation of cyclin D1 nuclear export and cyclin D1-dependent cellular transformation. Genes Dev. 2000, 14, 3102-3114.

23. Baldin, V.; Lukas, J.; Marcote, M.J.; Pagano, M.; Draetta, G. Cyclin D1 is anuclear protein required for cell cycle progression in G1. Genes Dev. 1993, 7, 812-821.

24. Shakoori, A.; Ougolkov, A.; Yu, Z.W.; Zhang, B.; Modarressi, M.H.; Billadeau, D.D.; Mai, M.; Takahashi, Y.; Minamoto, T. Deregulated GSK3 beta activity in colorectal cancer: Its association with tumor cell survival and proliferation. Biochem. Biophys. Res. Commun. 2005, 334, 1365-1373.

25. Doble, B.W.; Woodgett, J.R. GSK-3: Tricks of the tradefor a multi-tasking kinase. J. Cell Sci. 2003, 116, 1175-1186.

26. Frame, S.; Cohen, P.; Biondi, R.M. A common phosphate binding site explains the unique substrate specificity of GSK3 and its inactivation by phosphorylation. Mol. Cell 2001, 7, 1321-1327.

27. Davis, M.F.; Vigil, D.; Campbell, S.L. Regulation of Ras proteins by reactive nitrogen species. Free Radic. Biol. Med. 2011, 51, 565-575.

28. Pagano, M.; Theodoras, A.M.; Tam, S.W.; Draetta, G.F. Cyclin D1-mediated inhibition of repair and replicative DNA synthesis inhuman fibroblasts. Genes Dev. 1994, 8, 1627-1639.

29. Wu, D.N; Pei, D.S.; Wang, Q.; Zhang, G.Y. Down-regulation of PTEN by sodium or thovanadate inhibits ASK1 activation via PI3-K/Akt during cerebral ischemia in rat hippocampus. Neurosci. Lett. 2006, 404, 98-102.

30. Pan, X.; Gong, N.; Zhao, J.; Yu, Z.; Gu, F.; Chen, J.; Sun, X.; Zhao, L.; Yu, M.; Xu, Z.; et al. Powerful beneficial effects of benfotiamine on cognitive impairment and beta-amyloid deposition in amyloid precursor protein/presenilin-1 transgenic mice. Brain 2010, 133, 1342-1351.

31. Chen, Y.Z.; Zhi, D.G. Ligand-protein inverse docking and its potential use in the computer search of protein targets of a small molecule. Proteins 2001, 43, 217-226.

(C) 2015 by the authors; licensee MDPI, Basel, Switzerland. This article is an open access article distributed under the terms and conditions of the Creative Commons Attribution license (http://creativecommons.org/licenses/by/4.0/). 